\title{
Nonsense-mediated mRNA decay factor Upf 2 exists in both the nucleoplasm and the cytoplasm
}

\author{
TAKANORI TATSUNO ${ }^{1}$, YUKA NAKAMURA ${ }^{1}$, SHAOFU MA ${ }^{1}$, \\ NAOHISA TOMOSUGI ${ }^{1,2}$ and YASUHITO ISHIGAKI ${ }^{1}$ \\ ${ }^{1}$ Medical Research Institute, Kanazawa Medical University; ${ }^{2}$ Medical Care Proteomics \\ Biotechnology Co., Ltd., Kahoku, Ishikawa 920-0293, Japan
}

Received March 19, 2015; Accepted April 20, 2016

DOI: $10.3892 / \mathrm{mmr} .2016 .5331$

\begin{abstract}
Upf2 protein predominantly localizes to the cytoplasmic fraction, and binds to the exon junction complex (EJC) on spliced mRNA. The present study aimed to determine the cellular site where the interaction between Upf 2 and EJC occurs. First, the cell lysate was fractionated into the cytoplasm and nucleoplasm, and western blotting to detect levels of Upf2 protein was performed. Upf2 was clearly detected in the cytoplasm and in the nucleoplasm. Secondly, immunostaining was performed, and the majority of Upf2 was detected in the cytoplasmic perinuclear region; a small quantity of Upf2 was detected in the intranuclear region. RNase treatment of the cells reduced the Upf 2 immunostained signal. The immunopurified fractions containing nuclear and cytoplasmic Upf2 also contained one of the EJC core factors, RBM8A. These results implied the existence of Upf 2 in the nucleoplasm and the cytoplasm, and it appeared to be involved in the construction of the mRNA complex. In order to verify the construction of Upf2-binding EJC in the nucleoplasm, an in situ proximity ligation assay was performed with anti-Upf2 and anti-RBM8A antibodies. These results demonstrated that their interaction occurred not only in the cytoplasmic region, but also in the intranuclear region. Taken together, these results suggested that Upf2 combines with EJC in both the cytoplasmic and the intranuclear fractions, and that it is involved in mRNA metabolism in human cells.
\end{abstract}

\section{Introduction}

Previously, it has been reported that the aberrant copy number variation $(\mathrm{CNV})$ of the human Upf 2 gene locus is associated with neural development disorders, based on a search for

Correspondence to: Professor Yasuhito Ishigaki, Medical Research Institute, Kanazawa Medical University, 1-1 Daigaku, Uchinada, Kahoku, Ishikawa 920-0293, Japan

E-mail: ishigaki@kanazawa-med.ac.jp

Key words: Upf2, exon junction complex, nucleoplasm, mRNA, nonsense-mediated mRNA decay
CNVs among patients with intellectual disabilities (1). Upf3b, the binding partner of Upf 2 , is also associated with similar developmental disorders, and this protein is one component of the exon junction complex (EJC) formed on mRNA (2). The EJC, and its associated proteins, are essential factors of the nonsense-mediated mRNA decay (NMD) pathway, and NMD has various effects on cellular function. Therefore, it is possible that a deficiency of them may lead to neuro-developmental disorders.

mRNA forms messenger ribonucleoprotein particles (mRNPs) with various factors and is exported from the nucleus to the cytoplasm following transcription. During these processes, various quality control systems are responsible for maintaining the integrity of the genetic information. One of these processes is NMD, which eliminates mRNA that contains premature termination codons (PTCs) (3-6). PTCs may arise from a variety of transcription errors, including nonsense mutations in the genome, and they often cause recessive inherited disorders. Regardless of their source, PTCs in one allele have the potential to produce deleterious C-terminal truncated proteins, and NMD is considered to suppress the expression of truncated proteins that possibly have dominant negative toxicity caused by competitive inhibition of wild-type-allele-derived proteins. During the processes of mRNA metabolism, almost all the mRNAs that are exported from the nucleus to the cytoplasm pass through a quality control process, so that those containing PTCs may be eliminated to effectively maintain the integrity of the genetic information by NMD.

The conventional NMD mechanism requires the presence of various factors, including the SMG-1-Upf1-eRF1-eRF3 (SURF) complex (formed with SMG1, Upf1 and eukaryotic release factors), Upf2, Upf3b, EJC, and others. The EJC comprises RBM8A (Y14), Magoh, eIF4A3 and other proteins, and this is formed on the mRNA via splicing, serving as a platform for the assembly of the NMD-associated complex (7-10) or other functional complexes. The complex formed comprising Upf1, Upf2 and Upf3b is well established $(4,11)$. Upf3b and Upf2 are reported to localize at the EJC and receive SURF when the ribosome encounters PTCs, as determined from biochemical experiments (12).

Immunostaining experiments have revealed that EJC and Upf3b predominantly localize in the nucleus, whereas Upf1 
predominantly localizes in the cytoplasm. On the other hand, although Upf 2 has a nuclear localization signal, Upf 2 is located predominantly in the cytoplasmic perinuclear region $(11,13)$. The interaction of NMD factors is well established (14-16), and NMD is considered to progress according to the following three steps: First, Upf3b and EJC bind to mRNA molecules via RNA splicing in the nucleus; subsequently, perinuclear Upf2 binds to Upf $3 b$ on the exporting mRNA; and finally, following the formation of the EJC-Upf2-mRNA complex, it interacts with the SURF complex through Upf1 on the ribosomes arrested at a PTC, resulting in the selective degradation of mRNAs with PTCs $(4,12)$. It has been reported that NMD occurs either in the cytoplasm or in a nuclear-associated manner when newly synthesized mRNA undergoes the first round of translation, a process known as pioneer round translation $(3,5,6,17-19)$. On the other hand, the inhibitory effect of endogenous expression of dominant-negative Upf proteins on NMD activity was assessed, and it was proposed that the interaction between the Upf complex and EJC occurs in the cytoplasm, rather than in a nucleus-associated manner (20). Therefore, although NMD occurs in the cytoplasm, the possibility of nuclear-associated NMD during the export of mRNPs still remains.

According to previously proposed models $(10,15,16), \mathrm{Upf} 2$ accumulates at the perinuclear region and forms a complex with EJC on the exporting mRNA outside of the nuclei. However, almost all models have been established solely on spatial observations, and therefore have not provided any information concerning the mechanism for Upf 2 localization. If only perinuclear Upf 2 proteins are involved in forming a complex with EJC, nuclear-associated NMD should take place close to the nucleus, perhaps immediately following export from the nuclear pore complex. Alternatively, if intranuclear Upf2 protein forms complexes with EJC in the nuclei, the mRNA-EJC-Upf 2 complex would appear in time for an approach by SURF and the ribosome, even in nuclei. Indeed, at present, no published studies have identified the location of endogenous Upf2-EJC complex formation.

In the present study, fractionation of cell lysates and series of immunostaining experiments were performed. Taken together, our study using a proximity ligation in situ assay has demonstrated that endogenous Upf 2 interacts with one of the EJC core factors, RBM8A, in the inner nucleus prior to mRNA export through the nuclear pore, and constructs the mRNA-protein complex.

\section{Materials and methods}

Cell culture. HeLa and A549 cells were maintained in Dulbecco's modified Eagle's medium (Sigma-Aldrich, St. Louis, MO, USA) supplemented with $10 \%$ fetal bovine serum and antibiotics (final concentration, 10,000 U/ml penicillin and $10 \mathrm{mg} / \mathrm{ml}$ streptomycin; Wako Pure Chemical Industries, Ltd., Osaka, Japan). The cells were allowed to adhere and proliferate for $\geq 24 \mathrm{~h}$ at $37^{\circ} \mathrm{C}$ in $5 \% \mathrm{CO}_{2}$ prior to the following experiments.

Cellular fractionation. The preparation of nucleoplasmic and cytoplasmic fractions was performed as previously described (21). NE-PER nuclear and cytoplasmic extraction reagent (Pierce, Thermo Fisher Scientific, Inc., Rockford, IL, USA) was used according to the manufacturer's protocol, and prepared fractions were denatured with $2 \mathrm{X}$ Laemmli Sample buffer (Bio-Rad Laboratories, Inc., Hercules, CA, USA) for western blot analysis.

Western blotting. The procedures for whole lysate preparation and western blot analysis have been described (22). Protein concentration of the lysates was measured by the Bradford method. In brief, denatured samples $(25 \mu \mathrm{g})$ were applied to $10 \%$ acrylamide gels. Following SDS-PAGE, gels were transferred to polyvinylidene difluoride membranes and blocked with $5 \%$ skim milk for $1 \mathrm{~h}$. Then, blocked membranes were subjected to antibody binding. The first antibodies were rabbit anti-human Upf 2 polypeptide antiserum (prepared in our laboratory), monoclonal mouse anti-human lamin $\mathrm{A} / \mathrm{C}$ antibody (1:1,000; cat. no. sc-7292; Santa Cruz Biotechnology, Inc., Santa Cruz, CA, USA) and monoclonal rabbit anti-human caspase 3 (1:1,000; cat. no. 9665; Cell Signaling Technology, Inc., Danvers, MA, USA). In addition, monoclonal mouse anti-RBM8A antibody was purchased from Sigma-Aldrich (1:500; 4C4 clone; cat. no. Y1253). $\beta$-actin (ACTB) was used as the reference protein, and was detected using mouse monoclonal anti $\beta$-actin antibody purchased from Sigma-Aldrich (1:5,000; AC15 clone; cat. no. A5441). Incubation with primary antibody were performed at $4^{\circ} \mathrm{C}$, overnight. Primary antibodies were detected with horseradish peroxidase-conjugated polyclonal goat anti-mouse (1:5,000; cat. no. P0447) or anti-rabbit (1:5,000; cat. no. P0448) secondary antibodies (Dako, Glostrup, Denmark).

Gene expression/knockdown. One day prior to the small interfering RNA (siRNA) transfection experiments, HeLa cells were seeded on to culture plates or dishes. The depletion of Upf2 was performed using stealth RNA interference (RNAi) molecules (HSS178005), and the knockdown of RBM8A was achieved using HSS115052; the two siRNAs were obtained from Invitrogen ${ }^{\mathrm{TM}}$; Thermo Fisher Scientific, Waltham, MA, USA). Two double-stranded molecules of the Stealth RNAi ${ }^{\circledR}$ negative control kit (Thermo Fisher Scientific) and medium GC duplex were used as the negative control. Transfections were performed using Lipofectamine ${ }^{\mathrm{TM}}$ RNAiMAX transfection reagent (Thermo Fisher Scientific), according to the manufacturer's protocol.

Immunostaining and observation of the cells. The full details of the procedure followed for immunostaining were previously described (23). Briefly, HeLa cells were washed three times with phosphate-buffered saline (PBS) and fixed at room temperature with $4 \%$ paraformaldehyde solution (Taab Laboratory Equipment Ltd, Aldermaston, Reading, UK) in PBS for $10 \mathrm{~min}$. After washing three times with PBS, the cells were treated with $0.2 \%$ Triton X-100 (Sigma-Aldrich)/PBS for $10 \mathrm{~min}$ at room temperature. Samples were washed three times with PBS. For the ribonuclease (RNase) treatment experiments, cells were fixed with cold ethanol for $10 \mathrm{~min}$ and treated with $10 \mathrm{ng} / \mathrm{ml}$ RNaseA solution (Qiagen $\mathrm{GmbH}$, Hilden, Germany) at $37^{\circ} \mathrm{C}$ for $30 \mathrm{~min}$, followed by three further washes with PBS. Blocking was performed with $1 \%$ bovine serum albumin (BSA; Wako Pure Chemical Industries, 
Ltd.) with gentle agitation for $30 \mathrm{~min}$ at room temperature. Following this procedure, the cells were incubated with the first antibodies, rabbit anti-human Upf2 polypeptide antiserum prepared in our laboratory), anti-human lamin A/C antibody (Santa Cruz Biotechnology, Inc.), mouse anti-RBM8A antibody purchased from Sigma-Aldrich (4C4 clone) or rabbit anti-RBM8A antibody (prepared in our laboratory) with gentle agitation for $2 \mathrm{~h}$ at room temperature. Binding of the first antibody was detected using Alexa Fluor 488or 594-conjugated secondary antibodies (Molecular Probes Life Technologies, Carlsbad, CA, USA) with gentle agitation at room temperature for $60 \mathrm{~min}$, and nuclei were stained with 4',6-diamidino-2-phenylindole (DAPI). After washing the cells with PBS three times, ProLong ${ }^{\circledR}$ Gold Antifade reagent (Invitrogen $^{\mathrm{TM}}$; Thermo Fisher Scientific) was used to prevent fading. Images were captured with either an Axiovert $200 \mathrm{M}$ inverted fluorescence microscope or an LSM 710 confocal point-scanning microscope (Carl Zeiss, München, Germany). ZEN 2008 SP1.1 software (Carl Zeiss) was used to analyze signal intensity.

Immunoprecipitation of Upf2. To confirm the association between mRNA and Upf $2, \mathrm{HeL}$ a cells were collected using a cell scraper and suspended in cold NET-2 buffer [50 mM Tris- $\mathrm{HCl}$ (pH 7.4), 300 mM NaCl, 0.05\% Igepal-CA630; Sigma-Aldrich]. For nuclear fractions, cells were treated with cNE-PER nuclear and cytoplasmic extraction reagent (Thermo Fisher Scientific, Inc.) and nuclear fractions were collected. Subsequently, nuclear fractions were suspended in cold NET-2 buffer. The samples were sonicated using the Vibra cell sonicator (Sonics \& Materials, Inc., Newtown, CT, USA), and the sonicated samples were centrifuged for $15 \mathrm{~min}$ at $15,000 \mathrm{x}$ g. The supernatant was treated with anti-Upf 2 antiserum and normal rabbit serum as the control. Following an incubation with agitation in a cold room, Protein G-conjugated agarose beads (Invitrogen $^{\mathrm{TM}}$; Thermo Fisher Scientific) were added to the samples, and the samples thus obtained were again incubated in a cold room. Following sufficient washing with NET-2 buffer, the beads were resuspended in Laemmli sample buffer (Bio-Rad Laboratories, Inc.) with 2-mercaptoethanol (Sigma-Aldrich). The sample was boiled, and the resultant sample buffer solution was processed for western blotting, which was performed as described above.

Detection of complex formation with a proximity ligation in situ assay. The direct observation of Upf2-EJC complex formation was performed using a Duolink ${ }^{\circledR}$ kit (Olink Bioscience, Uppsala, Sweden; product now owned by Sigma-Aldrich) following the manufacturer's protocol (23). This method enables the visualization of complex formation in cells by proximity ligation of single-stranded DNA conjugated with a secondary antibody (24), and is available for the complex in nuclei and cytoplasm (25). Signal can be observed when the distance between the secondary antibodies is $<40 \mathrm{~nm}$. Therefore, this method can detect not only direct protein-protein interactions, but also complex formation, assuming that the bound first antibodies are proximal enough. The first antibodies used in the present study were as described in the above Immunostaining and observation section. Images were captured with either an Axiovert

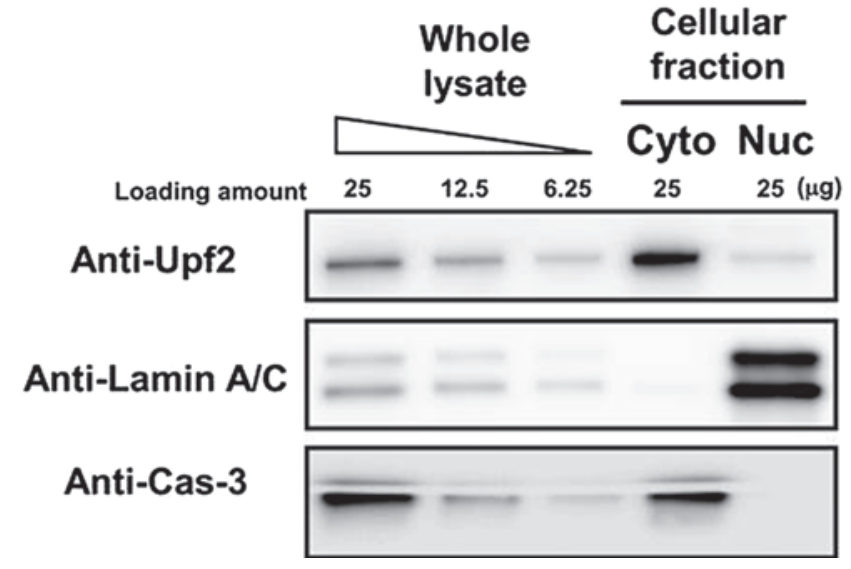

Figure 1. Detection of Upf2 protein in the cytoplasm and in the nucleoplasm. HeLa cells were fractionated, and the proteins were separated by sodium dodecyl sulfate-polyacrylamide gel electrophoresis, followed by western blotting. 'Cyto' shows the cytoplasmic fraction, and 'Nuc' shows the nucleoplasmic fraction. The membrane was blotted using anti-Upf2, anti-lamin A/C and anti-caspase 3 antibodies. Whole cell lysate was used as the control.

$200 \mathrm{M}$ inverted fluorescence microscope or an LSM 710 confocal point-scanning microscope (Carl Zeiss, München, Germany).

\section{Results}

Detection of Upf 2 by western blotting in the nuclear fraction. In order to assess whether Upf 2 was resident in the nuclei, HeLa cells were fractionated into their respective nuclear and cytoplasmic fractions. These fractions were analyzed using western blotting. The purity of each fraction was evaluated by blotting with anti-lamin A/C (nuclear marker) and anti-caspase 3 (cytoplasmic marker) antibodies. Almost all the lamin $\mathrm{A} / \mathrm{C}$ was detected in the nuclear fraction, and caspase 3 was predominantly detected in the cytoplasm. Therefore, it was confirmed that the fractionation process had been successful, and Upf2 was clearly detected in the two fractions (Fig. 1).

Detection of Upf 2 and RBM8A by immunostaining. Subsequently, to confirm the results of western blotting, immunostaining experiments were performed. Previous reports have revealed the cytoplasmic and perinuclear localization of endogenous or exogenous Upf2 $(11,13)$. In accordance with these reports, the results in the present study also demonstrated that Upf 2 is predominantly localized to the cytoplasmic perinuclear region, although a limited quantity of signal was observed in the nucleus (Fig. 2A). Subsequently, knockdown experiments were performed to confirm the specificity of the obtained signals. The Upf2-specific siRNA, prepared in our own laboratory, successfully depleted the Upf 2 signals in the perinuclear and intranuclear regions (Fig. 2A). The knockdown efficiency was confirmed by western blotting of the siRNA-treated and control siRNA-treated cells (Fig. 2B). These findings suggested that the signals from immunostaining observed in the present study had originated from the Upf2 molecules. Thus, it was deduced that Upf 2 proteins exist not only in the perinuclear region, but also in the nucleoplasmic region. 


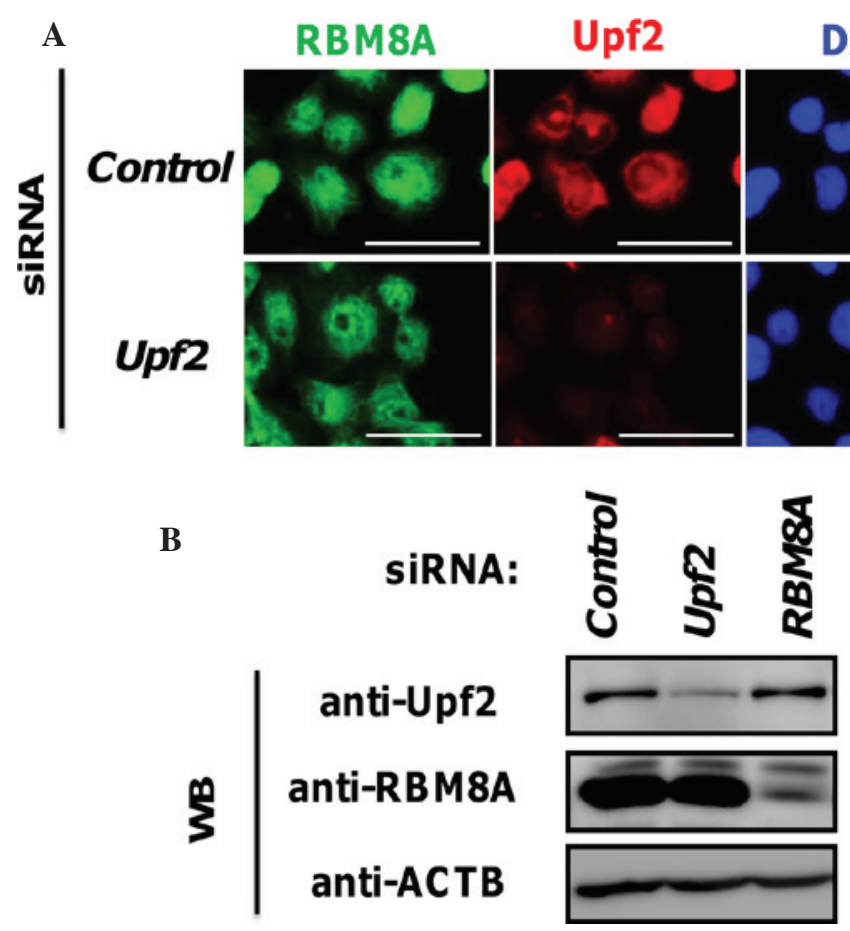

Figure 2. Detection of nuclear Upf2 by immunostaining. (A) Following transfection with siRNA in HeLa cells, the localization of Upf2 was analyzed using immunostaining. Fixed cells were treated with anti-Upf2 and anti-RBM8A antibodies followed by appropriate secondary antibodies. Nuclei were detected using DAPI staining. (B) The efficiency of knockdown was confirmed by western blotting. Bar=50 $\mu \mathrm{m}$. WB, western blot; siRNA, small interfering RNA; DAPI, 4',6-diamidino-2-phenylindole; ACTB, $\beta$-actin.

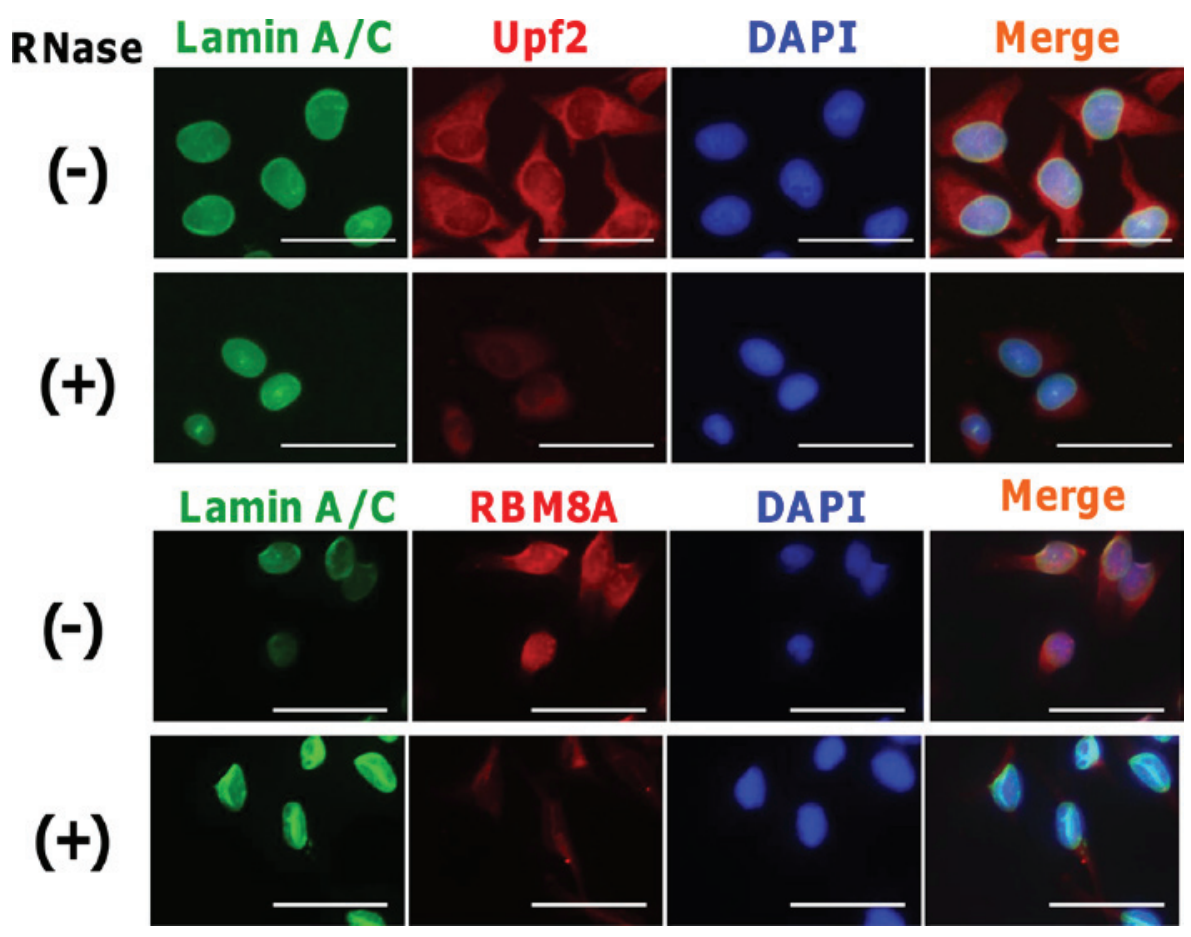

Figure 3. Effect of RNase treatment on Upf2 localization. Immunostaining of mouse anti-lamin A/C antibody, rabbit anti-Upf2 and anti-RBM8A antiserum with or without RNase treatment. Methanol-fixed cells were treated with RNaseA solution. Following washing, specimens were immunostained as described in the Materials and methods. Nuclei were detected using DAPI staining. Bar=50 $\mu \mathrm{m}$. RNase, ribonuclease; DAPI, 4',6-diamidino-2-phenylindole.

Upf 2 binds to RNA complex. To analyze which factors bind to the RNA molecule, RNase treatments are available and have been used in a number of previous studies (26-28). To examine whether Upf2 protein binds to RNA molecules in cells, methanol-fixed cells were treated with RNaseA solution. Following washing of the cells, cells were stained with anti-lamin A/C and anti-Upf 2 antibodies, as described in the Materials and methods section. In addition, RBM8A was also stained as a control for the RNase treatment. As shown in Fig. 3, the majority of Upf 2 in the cytoplasm and the nucleoplasm 


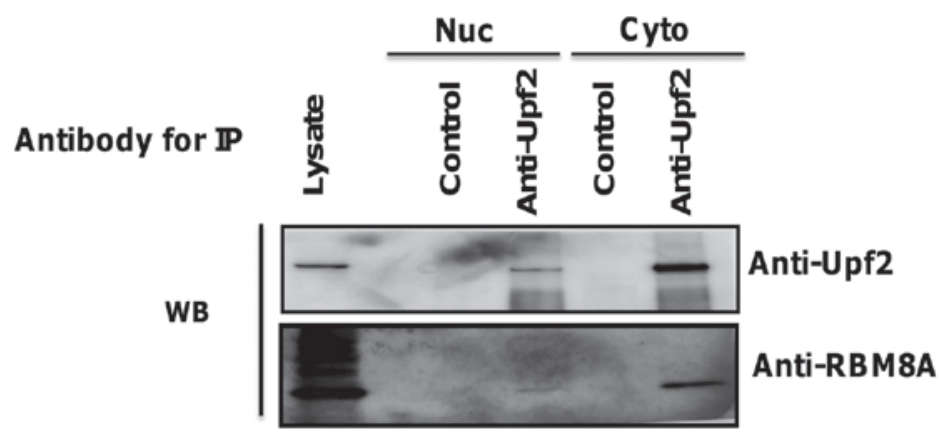

Figure 4. Upf 2 binds to mRNA in the nucleoplasm and the cytoplasm. Following fractionation of the cell lysate, immunopurification with anti-Upf2 antiserum was performed. As a control, rabbit preimmune serum was used. WB, western blot; IP, immunoprecipitation; Nuc, nuclear; Cyto, cytoplasmic.

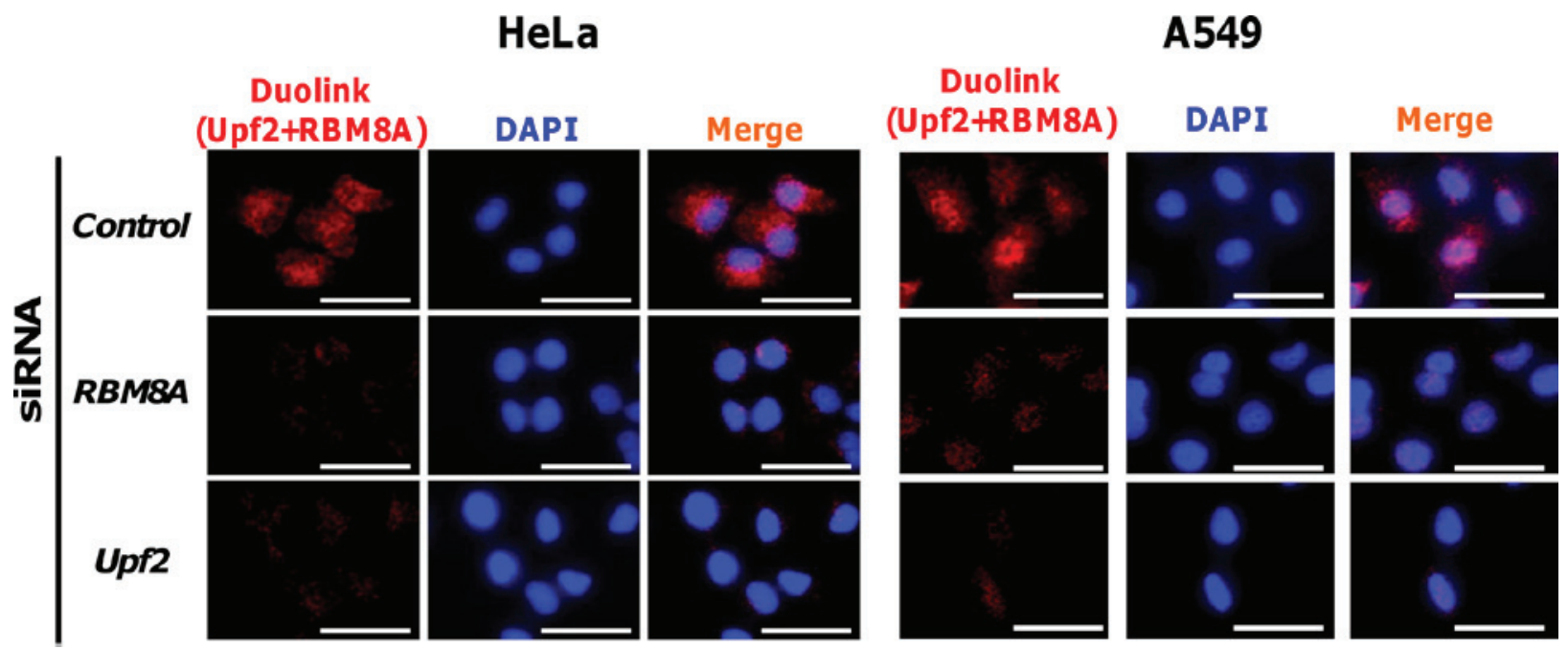

Figure 5. Knockdown of Upf2 or RBM8A reduced the signal intensity, as determined by a proximity ligation in situ assay. HeLa and A549 cells were treated with anti-Upf 2 and anti-RBM8A antibodies together, and their proximity was assessed using a DuoLink kit, as described in the Materials and methods section. Samples were treated with both anti-RBM8A and anti-Upf2 antibodies. Nuclei were detected by DAPI staining. Bar=50 $\mu$ m. siRNA, small interfering RNA; DAPI, 4',6-diamidino-2-phenylindole.

disappeared on RNase treatment. This suggested that appreciable quantities of Upf2 bind to the mRNA molecules in the nucleoplasm, as found for the cytoplasm and the perinuclear region. The signal from lamin $\mathrm{A} / \mathrm{C}$ remained, and this clearly demonstrated that the structural integrity of the nucleus was not affected by the RNase treatment.

The presence of Upf2-binding RBM8A in the nuclear fraction was subsequently investigated. Following fractionation, Upf2 protein was immunopurified using a specific antibody. RBM8A was detected to a limited extent in the immunoprecipitate of the nuclear fraction, similarly to the cytoplasmic fraction (Fig. 4).

Detection of complex formation between Upf2 and RBM8A using a proximity ligation in situ assay. Finally, to verify the interaction between Upf 2 and EJC in the nucleoplasm, a proximity ligation in situ assay with rabbit anti-Upf 2 and mouse anti-RBM8A antibodies (a component of EJC) was performed (7-10,29). Signals from the proximity ligation in situ assay were detected under a fluorescence microscope from the nuclei and the cytoplasm. In addition, knockdown of either the Upf2 or the RBM8A gene resulted in a reduction in signal intensity (Fig. 5). Under a confocal laser scanning microscope, sliced images were obtained, and the images revealed the nuclear-localized signals in addition to cytoplasmic signals (data not shown). These findings were not cell-type-specific, since similar results were obtained with human A549 cells under identical conditions. These results suggested that the Upf2 protein resides proximally to RBM8A in the nuclei and cytoplasm, and is included in the EJC.

\section{Discussion}

Previous reports have demonstrated that Upf2 binding at the perinuclear region efficiently promotes NMD before translation. Although Upf2 has a putative nuclear localization signal (NLS) sequence and is localized to the perinuclear region, whether Upf 2 is present in the nucleus remains unclear. Thus, the current study investigated the presence of Upf 2 in the nucleus. The data suggested that nuclear Upf 2 co-localizes with mRNPs in the nucleus. Thus. the previously proposed model $(10,15,16)$, which included cytoplasmic binding, requires the addition of a nucleoplasmic fraction.

Taken together, our results suggest that nuclear Upf2 co-localizes with mRNPs in the nucleus. The previously 
proposed model $(10,15,16)$, which included cytoplasmic binding, therefore requires the addition of a nucleoplasmic fraction. Since Upf2-associated NMD occurs in the cytoplasm (20), nuclear complex formation may not be associated with the cytoplasmic NMD reaction. In addition, the distribution of the Duolink signal did not perfectly correlate with the localization of Upf2, and complex formation and cytoplasmic Upf2 are able to exist without complex formation with EJC. Therefore, the mechanism that would account for the binding of Upf2 to the EJC in the nucleus has yet to be elucidated. Essentially, the molecular function of Upf2 has not been firmly established, other than the requirement for NMD activity. Since NMD occurs in the cytoplasm (20), given its nuclear function, additional functions for Upf 2 may be assumed. In a transcriptome analysis, depletion of Upf2 was demonstrated to cause physiological changes in various genes without PTC (30). Therefore, Upf 2 may have additional functions that would be required for the proper development of the human neural system. Additionally, the quantity of Upf2 that resides in the nuclei has yet to be fully established, since there is a possibility that the western blots and immunoprecipitation data also contained nuclear-flanked Upf2. Therefore, further investigations are revealed to reveal additional functions of Upf2, including its role in the nucleus.

\section{Acknowledgements}

This work was supported by grants from the Kanazawa Medical University (nos. C2014-3, S2014-15), a grant from the Strategic Research Project (no. H2012-16 [S1201022]), from Kanazawa Medical University, Ministry of Education, Culture, Sports, Science and Technology, Japan (KAKENHI no. \#25460376) and from the Sumitomo Science Foundation in Japan.

\section{References}

1. Nguyen LS, Kim HG, Rosenfeld JA, Shen Y, Gusella JF, Lacassie Y, Layman LC, Shaffer LG and Gécz J: Contribution of copy number variants involving nonsense-medated mRNA decay pathway genes to neuro-developmental disorders. Hum Mol Genet 22: 1816-1825, 2013.

2. Tarpey PS, Raymond FL, Nguyen LS, Rodriguez J, Hackett A, Vandeleur L, Smith R, Shoubridge C, Edkins S, Stevens C, et al: Mutations in UPF3B, a member of the nonsense-mediated mRNA decay complex, cause syndromic and nonsyndromic mental retardation. Nat Genet 39: 1127-1133, 2007.

3. Bühler M, Wilkinson MF and Mühlemann O: Intranuclear degradation of nonsense codon-containing mRNA. EMBO Rep 3: 646-651, 2002.

4. Chamieh H, Ballut L, Bonneau F and Le Hir H: NMD factors UPF2 and UPF3 bridge UPF1 to the exon junction complex and stimulate its RNA helicase activity. Nat Struct Mol Biol 15: 85-93, 2008.

5. Mühlemann O, Eberle AB, Stalder L and Zamudio Orozco R: Recognition and elimination of nonsense mRNA. Biochim Biophys Acta 1779: 538-549, 2008

6. Maquat LE, Tarn WY and Isken O: The pioneer round of translation: Features and functions. Cell 142: 368-374, 2010.

7. Gehring NH, Neu-Yilik G, Schell T, Hentze MW and Kulozik AE: Y14 and hUpf3b form an NMD-activating complex. Mol Cell 11: 939-949, 2003.

8. Gehring NH, Lamprinaki S, Hentze MW and Kulozik AE: The hierarchy of exon-junction complex assembly by the spliceosome explains key features of mammalian nonsense-mediated mRNA decay. PLoS Biol 7: e1000120, 2009.

9. Kataoka N, Yong J, Kim VN, Velazquez F, Perkinson RA, Wang F and Dreyfuss G: Pre-mRNA splicing imprints mRNA in the nucleus with a novel RNA-binding protein that persists in the cytoplasm. Mol Cell 6: 673-682, 2000.
10. Kim VN, Kataoka N and Dreyfuss G: Role of the nonsense-mediated decay factor hUpf 3 in the splicing-dependent exon-exon junction complex. Science 293: 1832-1836, 2001.

11. Serin G, Gersappe A, Black JD, Aronoff R and Maquat LE: Identification and characterization of human orthologues to Saccharomyces cerevisiae Upf 2 protein and Upf3 protein (Caenorhabditis elegans SMG-4). Mol Cell Biol 21: 209-223, 2001.

12. Kashima I, Yamashita A, Izumi N, Kataoka N, Morishita R, Hoshino S, Ohno M, Dreyfuss G and Ohno S: Binding of a novel SMG-1-Upf1-eRF1-eRF3 complex (SURF) to the exon junction complex triggers Upf1 phosphorylation and nonsense-mediated mRNA decay. Genes Dev 20: 355-367, 2006.

13. Lykke-Andersen J, Shu MD and Steitz JA: Human Upf proteins target an mRNA for nonsense-mediated decay when bound downstream of a termination codon. Cell 103: 1121-1131, 2000.

14. Kim VN, Yong J, Kataoka N, Abel L, Diem MD and Dreyfuss G: The Y14 protein communicates to the cytoplasm the position of exon-exon junctions. EMBO J 20: 2062-2068, 2001.

15. Le Hir H, Gatfield D, Izaurralde E and Moore MJ: The exon-exon junction complex provides a binding platform for factors involved in mRNA export and nonsense-mediated mRNA decay. EMBO J 20: 4987-4997, 2001.

16. Schell T, Kulozik AE and Hentze MW: Integration of splicing, transport and translation to achieve mRNA quality control by the nonsense-mediated decay pathway. Genome Biol 3: REVIEWS1006, 2002.

17. Ishigaki Y, Li X, Serin G and Maquat LE: Evidence for a pioneer round of mRNA translation: mRNAs subject to nonsense-mediated decay in mammalian cells are bound by CBP80 and CBP20. Cell 106: 607-617, 2001.

18. Chang YF, Imam JS and Wilkinson MF: The nonsense-mediated decay RNA surveillance pathway. Annu Rev Biochem 76: 51-74, 2007.

19. Maquat LE: Nonsense-mediated mRNA decay: Splicing, translation and mRNP dynamics. Nat Rev Mol Cell Biol 5: 89-99, 2004.

20. Singh G, Jakob S, Kleedehn MG and Lykke-Andersen J: Communication with the exon-junction complex and activation of nonsense-mediated decay by human Upf proteins occur in the cytoplasm. Mol Cell 27: 780-792, 2007.

21. Zhao X, Nogawa A, Matsunaga T, Takegami T, Nakagawa H and Ishigaki Y: Proteasome inhibitors and knockdown of SMG1 cause accumulation of Upf1 and Upf2 in human cells. Int J Oncol 44: 222-228, 2014

22. Ishigaki Y, Nakamura Y, Tatsuno T, Hashimoto M, Shimasaki T, Iwabuchi K and Tomosugi N: Depletion of RNA-binding protein RBM8A (Y14) causes cell cycle deficiency and apoptosis in human cells. Exp Biol Med (Maywood) 238: 889-897, 2013.

23. Ishigaki Y, Nakamura Y, Tatsuno T, Hashimoto M, Iwabuchi K and Tomosugi N: RNA binding protein RBM8A (Y14) and MAGOH localize to centrosome in human A549 cells. Histochem Cell Biol 141: 101-109, 2014.

24. Söderberg O, Gullberg M, Jarvius M, Ridderstråle K, Leuchowius KJ, Jarvius J, Wester K, Hydbring P, Bahram F, Larsson LG and Landegren U: Direct observation of individual endogenous protein complexes in situ by proximity ligation. Nat Methods 3: 995-1000, 2006.

25. Hervouet E, Lalier L, Debien E, Cheray M, Geairon A, Rogniaux H, Loussouarn D, Martin SA, Vallette FM and Cartron PF: Disruption of Dnmt1/PCNA/UHRF1 interactions promotes tumorigenesis from human and mice glial cells. PLoS One 5: e11333, 2010.

26. Spector DL, Fu XD and Maniatis T: Associations between distinct pre-mRNA splicing components and the cell nucleus. EMBO J 10: 3467-3481, 1991.

27. den Engelsman J, Bennink EJ, Doerwald L, Onnekink C, Wunderink L, Andley UP, Kato K, de Jong WW and Boelens WC: Mimicking phosphorylation of the small heat-shock protein alphaB-crystallin recruits the F-box protein FBX4 to nuclear SC35 speckles. Eur J Biochem 271: 4195-4203, 2004.

28. Zirwes RF, Eilbracht J, Kneissel S and Schmidt-Zachmann MS: A novel helicase-type protein in the nucleplus: Protein NOH61. Mol Cell Biol 11: 1153-1167,2000.

29. Schmidt $\mathrm{U}$, Richter $\mathrm{K}$, Berger $\mathrm{AB}$ and Lichter $\mathrm{P}$ : In vivo $\mathrm{BiFC}$ analysis of Y14 and NXF1 mRNA export complexes: Preferential localization within and around SC35 domains. J Cell Biol 172: 373-381, 2006.

30. Wittmann J, Hol EM and Jäck HM: hUPF2 silencing identifies physiologic substrates of mammalian nonsense-mediated mRNA decay. Mol Cell Biol 26: 1272-1287, 2006. 